\title{
Resonant tunneling diode oscillators for optical communications
}

Scott Watson

Weikang Zhang

Jue Wang

Abdullah Al-Khalidi

Horacio Cantu

Jose Figueiredo

Edward Wasige

Anthony E. Kelly 


\title{
Resonant tunneling diode oscillators for optical communications
}

\author{
Scott Watson*a, Weikang Zhang ${ }^{\mathrm{a}}$, Jue Wang ${ }^{\mathrm{a}}$, Abdullah Al-Khalidi ${ }^{\mathrm{a}}$, Horacio Cantu ${ }^{\mathrm{b}}$, Jose \\ Figueiredo $^{c}$, Edward Wasige ${ }^{a}$ and Anthony E. Kelly ${ }^{\mathrm{a}}$ \\ ${ }^{a}$ School of Engineering, University of Glasgow, Oakfield Avenue, Glasgow, G12 8LT, UK. \\ ${ }^{\mathrm{b}}$ Compound Semiconductor Technologies Global, Hamilton International Technology Park, 4 \\ Stanley Blvd, Blantyre, Glasgow, G72 0BN, UK. \\ ${ }^{c}$ Departamento de Fisica, Universidade do Algarve, Campus de Gambelas, 8005-139 Faro, Portugal.
}

\begin{abstract}
The ability to use resonant tunneling diodes (RTDs) as both transmitters and receivers is an emerging topic, especially with regards to wireless communications. Successful data transmission has been achieved using electronic RTDs with carrier frequencies exceeding $0.3 \mathrm{THz}$. Specific optical-based RTDs, which act as photodetectors, have been developed by adjusting the device structure to include a light absorption layer and small optical windows on top of the device to allow direct optical access. This also allows the optical signal to directly modulate the RTD oscillation. Both types of RTD oscillators will allow for seamless integration of high frequency radio and optical fiber networks.
\end{abstract}

Keywords: Resonant tunneling diodes, optical communications, modulation, terahertz sources, photodetector.

\section{INTRODUCTION}

Semiconductor photodetectors are widely used in optical communication systems to convert optical signals into electrical signals. There are many traditional types of photodetector used in fiber optic systems such as p-i-n photodiodes and avalanche photodiodes [1]. Optically controlled microwave devices have also been investigated such as Gunn diode oscillators and impact avalanche and transit time (IMPATT) oscillators, however due to their bandwidth limitations, requirements for high bias voltage and bulky cooling systems, other options need to be considered for high volume commercial applications. Resonant tunneling diode (RTD) oscillators which can operate at terahertz frequencies have been realized and are becoming a leading source for wireless communications due to their compact nature and efficiency [2], [3]. RTDs are the fastest electronic devices and have the capability to operate in the terahertz region at room temperature, making them ideal candidates to be incorporated into existing wireless or fiber optic networks. In addition to this, RTDs can also be exploited as photodetectors, which means they can be used as both transmitters and receivers in a communications system. A potential scenario making use of these RTDs as photodetectors can be seen in Figure 1. With the use of an integrated antenna, the signal received by the RTD can then be transmitted wirelessly using terahertz frequencies for the uplink (UL) or alternatively can be used as a terahertz receiver on the downlink (DL).

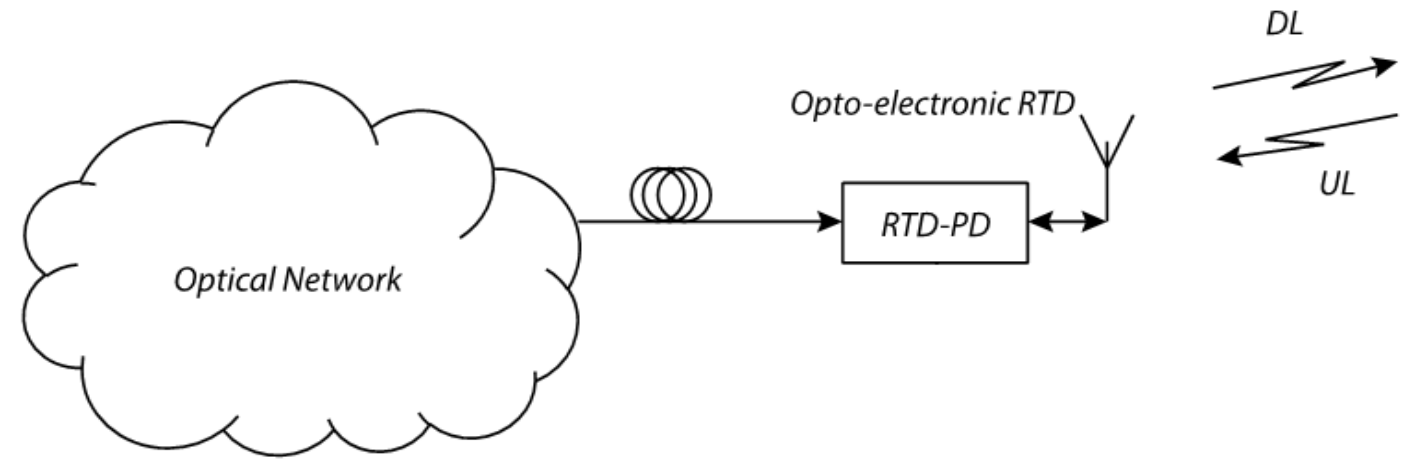

Figure 1. Potential application scenario using these RTDs as a photodetector.

*scott.watson@glasgow.ac.uk; phone +44 141 330-8443

Third International Conference on Applications of Optics and Photonics, edited by Manuel F. M. Costa, Proc. of SPIE Vol. 10453, 104532Q - @ 2017 SPIE · CCC code: 0277-786X/17/\$18 · doi: 10.1117/12.2276319 
By taking advantage of the nature of the non-linear I-V characteristics which shows negative differential resistance (NDR), detection of gigahertz modulated signals can be achieved successfully. There is also the prospect of driving an array of these RTDs with one, master optical signal to further increase capability. This paper will convey recent measurements carried out with these RTDs acting as photodetectors. Data transmission experiments have been conducted using non-oscillating RTD devices and the performance of RTD oscillators is analyzed in terms of oscillation frequency and it's response to modulated data.

\subsection{RTD structure and design}

An RTD is a unipolar, two terminal semiconductor with an N-shaped I-V characteristic at room temperature, exhibiting controllable NDR regions which provide electrical gain allowing high frequency oscillators to be implemented. RTDs are fabricated by having a narrow bandgap material sandwiched between two, thin, wide bandgap materials. This makes up the double barrier quantum well (DBQW) structure. For the oscillators discussed here, the epi-layer structure was grown by molecular beam epitaxy (MBE) by IQE Ltd on a semi-insulating InP substrate. Vertical stacking of nanometric scale epi-layers comprising a $5.7 \mathrm{~nm}$ undoped indium gallium arsenide (InGaAs) layer sandwiched between two $1.7 \mathrm{~nm}$ aluminium arsenide (AlAs) barrier layers make up the DBQW. This is surrounded by low doped $500 \mathrm{~nm}$ InGaAlAs/InGaAs spacer epi-layers which act as light absorbing regions where photo-generated electron-hole pairs are created upon incident light.

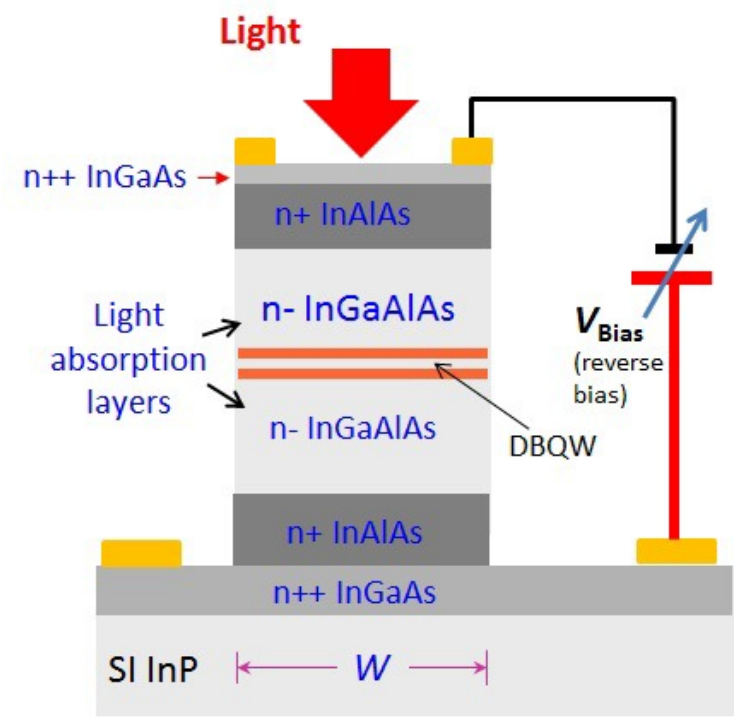

Figure 2. Layer structure of an RTD which is being used as a photodetector.

The RTD structure is completed with very highly doped InGaAs layers for ohmic contact formation. The RTD device size is $10 \times 10 \mu \mathrm{m}^{2}$, with an optical window of $10 \times 5 \mu \mathrm{m}^{2}$, however other device sizes and window sizes have been fabricated. The estimated responsivity of typical devices of this size at $1310 \mathrm{~nm}$ and $1550 \mathrm{~nm}$ are $0.2 \mathrm{~A} / \mathrm{W}$ and $0.07 \mathrm{~A} / \mathrm{W}$, respectively. The RTD oscillator circuits employ two RTD devices in parallel connected to a shorted $50 \Omega$ coplanar waveguide (CPW) line which provides the inductance required to resonate with the combined RTD selfcapacitances for a given targeted oscillation frequency. Measurements with both non-oscillating RTD devices and RTD oscillators will be discussed in the following sections.

\section{EXPERIMENTAL RESULTS}

\subsection{RTD device measurements}

As seen above, these RTD-based photodetectors (RTD-PDs) are nano-electronic structures consisting of vertical stacking of semiconductor epitaxial layers. The active region includes a typical DBQW structure which has a photoconductive region within which photon-generated electron-holes are created when illuminated with light. It works like a Fabry-Perot 
interferometer that has a non-linear and N-shaped voltage-current relationship i.e. the NDR region. When the RTD is biased in this region, the incident modulated optical signal induces photocharges which reduces the series resistance of the device due to inter-band absorption, and therefore shifts the peak and valley voltages leading to current oscillations emulating the RF signal [4].

An RTD with a device size of $10 \times 10 \mu \mathrm{m}^{2}$ which has an optical window of $10 \times 5 \mu \mathrm{m}^{2}$ was used for initial characterization experiments. The optical window offers a pathway for the incident signal to reach the photoconductive region. Both $1310 \mathrm{~nm}$ and $1550 \mathrm{~nm}$ wavelengths of light have been tested with similar performance. Figure 3 shows the change in the I-V plot, comparing the scenario with no light and illumination of different power levels at a wavelength of $1550 \mathrm{~nm}$. The peak current is around $-9.4 \mathrm{~mA}$ which occurs at a bias voltage of $-1.125 \mathrm{~V}$. As the illumination power level increases, the peak current increases slightly and shifts to a lower voltage. This shift based on the injection of light is due to the built-in electrical field close to the collector barrier which is enhanced due to the accumulation of lightgenerated holes in the collector absorption layer [5].

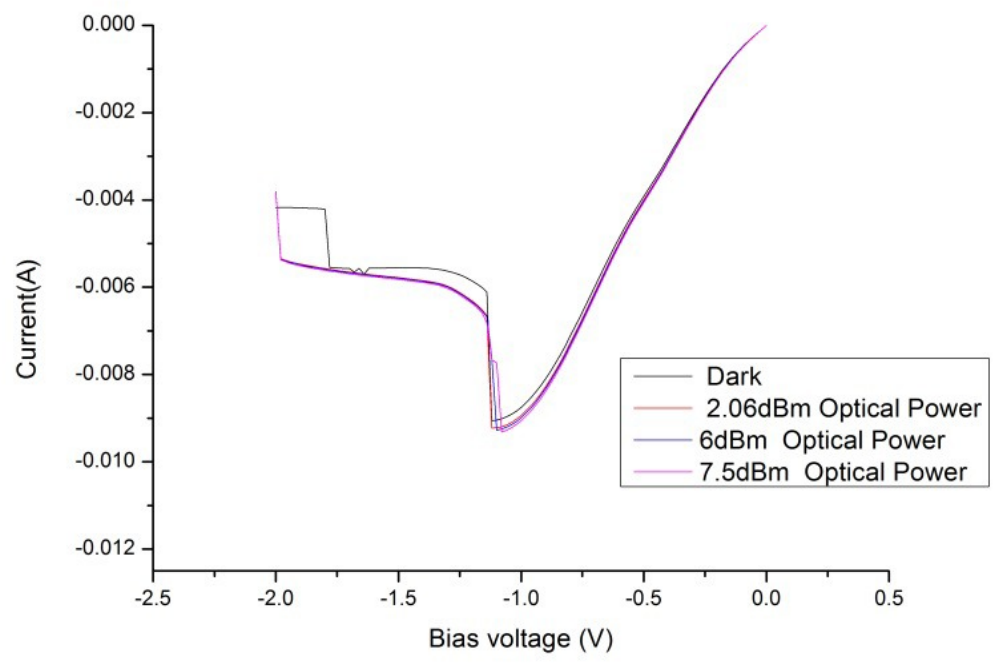

Figure 3. RTD I-V measurement at different input powers of light.

Frequency response and data transmission experiments were then conducted with this RTD-PD using the experimental setup shown in Figures 4(a) and 4(b), respectively. A negative bias was applied to the device, i.e. when using a groundsignal-ground (G-S-G) probe, the center pin going to the device was negative with respect to the ground connection. Light from a $1310 \mathrm{~nm}$ laser was combined with an RF signal from a network analyzer using a modulator and this modulated signal was sent to the RTD-PD via some lensed fiber. The signal was then taken off the RTD-PD using a high speed probe and sent back to the network analyzer electrically to view the frequency response of the device. A $-3 \mathrm{~dB}$ bandwidth of approximately $700 \mathrm{MHz}$ was measured for this device. The network analyzer was then changed for a biterror rate tester (BERT) where a pseudo-random bit sequence was combined with the light and used to modulate the RTD-PD. Again, the signal was taken off the device using a high speed probe and was connected to an oscilloscope in order to view the results in the form of eye diagrams. An eye diagram at a data rate of $250 \mathrm{Mbit} / \mathrm{s}$ is shown which has a signal to noise ratio (SNR) of 10.33. A useful response was observed up to $1 \mathrm{Gbit} / \mathrm{s}$ which can be seen in Figure 5. The reduction in eye opening is consistent with the measured bandwidth. 

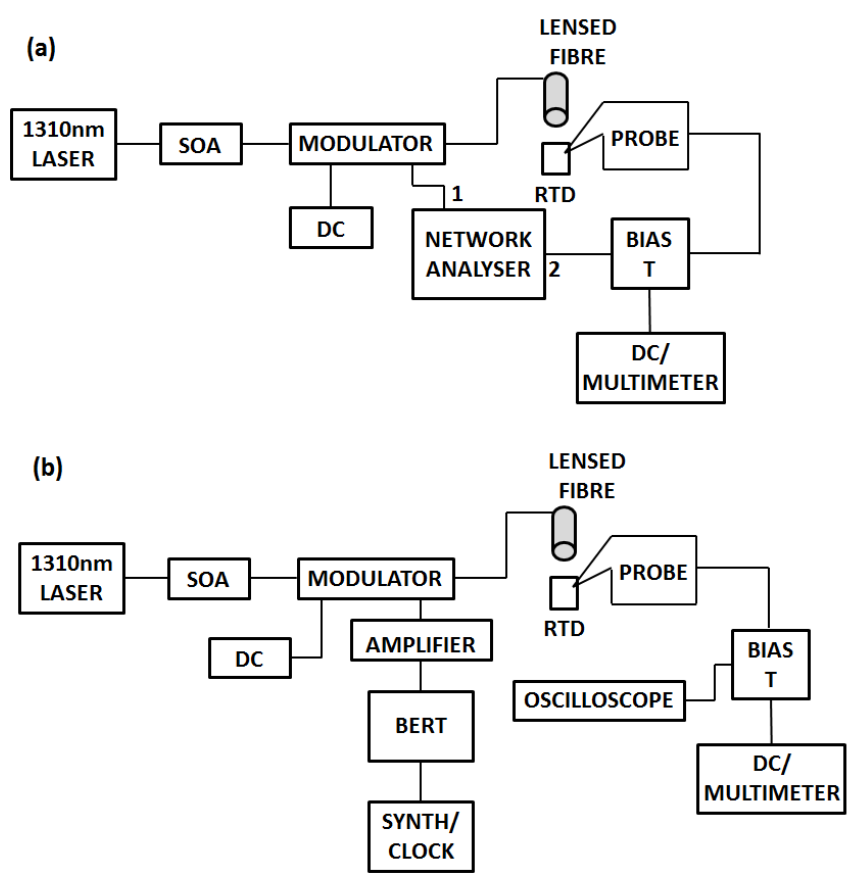

Figure 4. Experimental setup to carry out (a) frequency response measurements and (b) data transmission measurements.
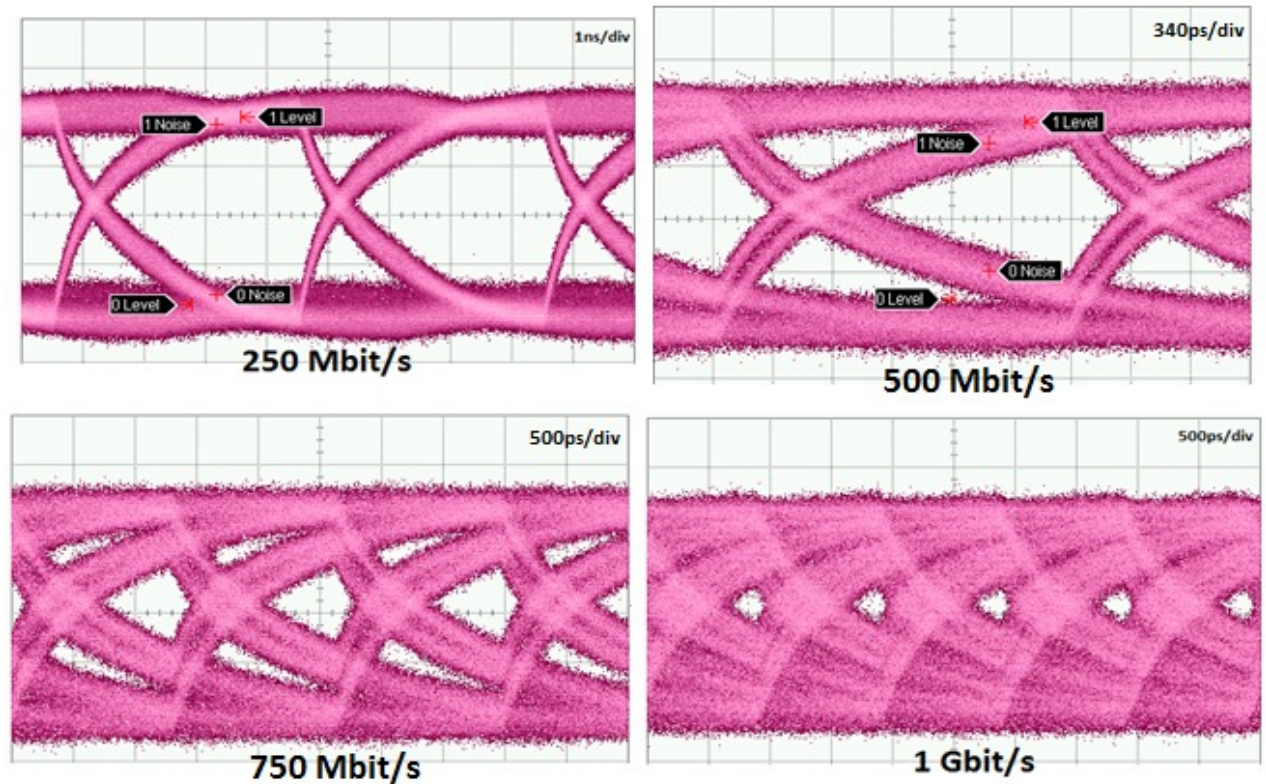

Figure 5. Eye diagrams showing data output achieved at $250 \mathrm{Mbit} / \mathrm{s}$ up to $1 \mathrm{Gbit} / \mathrm{s}$.

\subsection{RTD oscillator measurements}

In order to get more power from the RTD-PD oscillators, some structures employ two RTDs in parallel. The difference between the devices mentioned in Section 2.1 and RTD oscillators here is the addition of an RF circuit which causes the RTD to resonate at the desired oscillation frequency. A typical I-V measurement is shown in Figure 6 . The variations seen on the right hand side of the graph were due to experimental error; however the NDR region can be seen clearly around $-1.4 \mathrm{~V}$. As before, it is possible to shift the NDR region based on the intensity of the light incident on the RTD$\mathrm{PD}$, as seen in Figure 7. This graph shows the effect whilst having positive bias applied to the oscillator; however the 
shift to the right is apparent. When the optical power was too high, no NDR region could be seen. One possible explanation is that a large number of electron-hole pairs are generated and the photo-generated holes accumulate at the interface of the double barrier structure and the absorption layer. However, subsequent holes may not accumulate at the interface and merely stay a distance away because of the repulsive force of the existing holes which have accumulated. The effect of band modulation caused by these holes becomes weaker with the increase of this distance between the accumulated holes and the interface. Therefore, only a small variation of the tunneling current can be triggered [6].

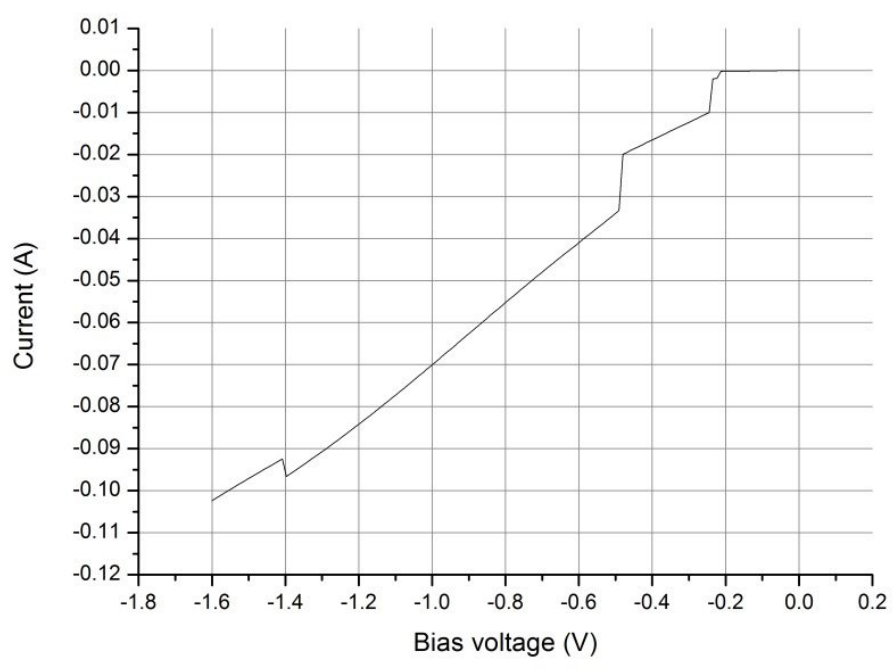

Figure 6. Typical I-V characteristics for a dual-RTD structure.

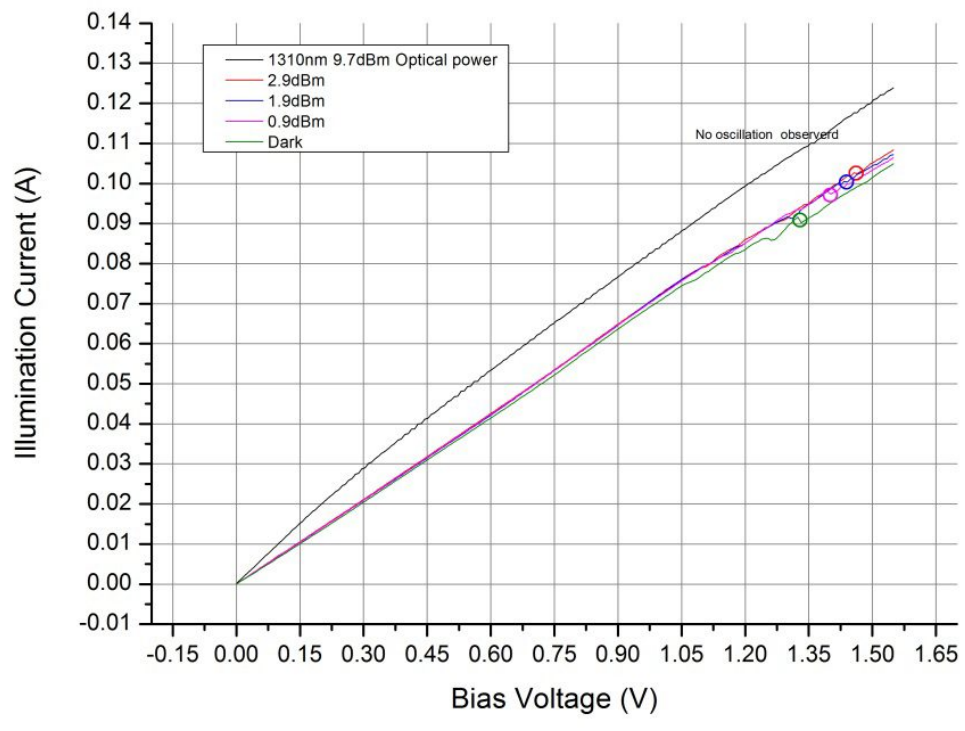

Figure 7. NDR region shifting based on increasing optical illumination intensity.

Data experiments were conducted, with the optical power set at $2.5 \mathrm{dBm}$ to ensure there is a working NDR region. Sidebands appear based on the optically injected data pattern. Figure 8 shows sidebands which are $1.25 \mathrm{GHz}$ apart from the oscillation frequency i.e. a data rate of $1.25 \mathrm{Gbit} / \mathrm{s}$. The amplitude of the sidebands with respect to the carrier will 
determine the data rate at which successful data transmission could be achieved, however sidebands could still be seen $5 \mathrm{GHz}$ away from the main carrier, showing that the prospect for faster transmission is promising.

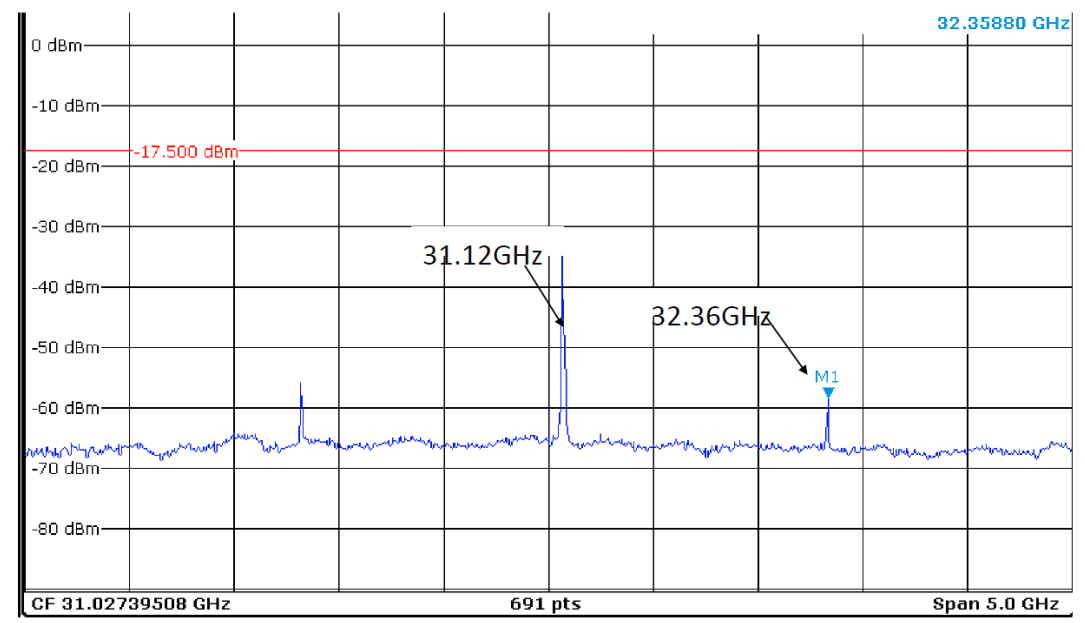

Figure 8. Spectrum showing oscillation frequency and sidebands based on an optically modulated data pattern at $1.25 \mathrm{Gbit} / \mathrm{s}$.

\section{CONCLUSIONS}

This paper illustrates the ability to use RTDs as photodetectors for optical communication systems. The ability to optically control such devices has been shown, with successful detection of modulated optical signals also presented. Data experiments have been carried out using an RTD as a photodetector which showed a bandwidth of approximately $700 \mathrm{MHz}$ and data transmission at $1 \mathrm{Gbit} / \mathrm{s}$. An analysis of the performance of dual-RTD oscillator structures was carried out before further data experiments were carried out using this RTD oscillator at $31.1 \mathrm{GHz}$. The ability to measure sidebands next to the oscillation frequency shows that modulation of the carrier is possible. These preliminary results indicate that RTD-PDs could provide useful functionality in Radio over Fiber (RoF) networks. These are potentially low cost and offer the possibility of bi-directional transmission.

\section{ACKNOWLEDGEMENTS}

This work was performed as part of the iBROW project (www.ibrow-project.eu) which has received funding from the European Union's Horizon 2020 research and innovation programme under grant agreement No. 645369.

\section{REFERENCES}

[1] E. Sackinger, Broadband circuits for optical fiber communication, John Wiley \& Sons, (2005).

[2] E. Wasige, K. H. Alharbi, A. Al-Khalidi, J. Wang, A. H. Khalid, G. C. Rodrigues and J. Figueiredo, "Resonant tunneling diode terahertz sources for broadband wireless communications," in SPIE Photonics West (OPTO), (2017).

[3] N. Oshima, K. Hashimoto, S. Suzuki and M. Asada, "Wireless data transmission of $34 \mathrm{Gbps}$ at a 500-GHz range using resonant tunneling diode oscillator," Electronics Letters, vol. 52, no. 22, (2016).

[4] T. Moise, Y. -C. Kao, C. Goldsmith, C. Schow and J. Campbell, "High speed resonant tunneling photodetectors with low switching energy," IEEE Photonics Technology Letters, vol. 9, no. 6, pp. 803-805, (1997).

[5] A. Pfenning, F. Hartmann, F. Langer, M. Kamp, S. Hofling and L. Worschech, "Sensitivity of resonant tunneling diode photodetectors," Nanotechnology, vol. 27, no. 35, p. 355202, (2016).

[6] Y. Dong, J. Xu, G. Wang, H. Ni, K. Pei, J. Chen, F. Gao, B. Li and Z. Niu, "Resonant tunneling diode photodetector operating at near-infrared wavelengths with high responsivity," Electronics Letters, vol. 15, no. 17, pp. 1355-1357, (2015). 